\title{
Consideraciones médico-odontológicas en la atención del niño con celulitis facial odontogénica. Una patología de interés para pediatras y odontopediatras \\ Medical-dental considerations in the care of children with facial cellulitis of odontogenic origin. A disease of interest for pediatricians and pediatric dentists
}

\author{
Od. Claudia Giunta Crescente ${ }^{a}$ Dra. Milagro Soto de Facchin ${ }^{a}$ y \\ Od. Andreína M. Acevedo Rodrígueza
}

\section{RESUMEN}

La infección odontogénica es una infección polimicrobiana y mixta (aerobios/anaerobios). Comprende diversos cuadros clínicos, cuya importancia deriva de su frecuencia y gravedad potencial. Es el tipo de infección más frecuenteen la cavidad bucal y su tratamiento supone hasta el $10 \%$ del total de prescripciones de antibióticos. La celulitis facial es una inflamación difusa delos tejidos blandos que no está circunscrita o limitada a una región y tiende a diseminarse. El objetivo de esta revisión es actualizar las consideraciones para la atención del niño con celulitis facial de origen dental y el manejo multidisciplinario entre el odontólogo y el pediatra.

Palabras clave: celulitis facial odontogénica, atención, niño.

http: / / dx.doi.org/10.5546/ aap.2018.e548 Texto completo en inglés:

http: / / dx.doi.org/10.5546/ aap.2018.eng.e548

Cómo citar: Giunta Crescente C, Soto de Facchin M, Acevedo Rodríguez AM. Consideraciones médicoodontológicas en la atención del niño con celulitis facial odontogénica. Una patología de interés para pediatras y odontopediatras. Arch Argent Pediatr 2018;116(4):e548-e553.

a. Facultad de Odontología, Universidad de Carabobo (UC), Venezuela.

Correspondencia: Od. Claudia Giunta: claudiagiunta3@ hotmail.com

Financiamiento: Ninguno.

Conflicto de intereses: Ninguno que declarar.

Recibido: 10-10-2017 Aceptado: 11-1-2018
La celulitis odontogénica es una inflamación aguda, profunda y difusa del tejido celular subcutáneo que se extiende por los espacios entre el tejido celular a más de una región anatómica, espacios tisulares y a lo largo de los planos aponeuróticos, a causa de la infección de uno o varios dientes o de patologías asociadas al tejido dentario o de sostén. ${ }^{2}$

Clínicamente, se manifiesta de forma muy variable, desde un proceso inocuo bien delimitado hasta un cuadro clínico progresivo y difuso, que puede ocasionar complicaciones. Cuando se palpa la celulitis en fase temprana, es de consistencia blanda, suave y con signos inflamatorios; los bordes del área inflamatoria son indefinidos y, algunas veces, no hay elevación de la epidermis subyacente. En la fase avanzada, el área afectada adquiere una consistencia indurada. ${ }^{2}$

Al momento del examen intrabucal, se evalúa el grado de apertura bucal, la cual se puede ver limitada por presencia de dolor y trismus. Entre las características clínicas, se puede observar borramiento del surco y movilidad o extrusión dentaria. ${ }^{2}$

Los síntomas generales de las infecciones de la región de la cabeza y el cuello son variables. La sepsis se manifiesta con apatía, debilidad, malestar, picos febriles, sudoración, pulso filiforme, leucocitosis $y$, en ocasiones, considerable anemia secundaria. El espasmo o 
inmovilización de los músculos contiguos origina trismo, tortícolis y rigidez. El compromiso neural provoca dolor en el nervio sensitivo afectado y parálisis de los nervios motores. También pueden ocurrir disfagia, disfonía y afonía, según el sitio de la infección. ${ }^{1}$

El tratamiento de la celulitis facial odontogénica en niños está en relación con las condiciones del estado general del paciente y el estado evolutivo del cuadro clínico. El control y la eliminación del agente causal es el objetivo principal, que, en algunos casos, requiere de la atención del médico especialista en pediatría para el tratamiento hospitalario del paciente. ${ }^{3}$

\section{Etiología}

La etiopatogenia de la celulitis facial odontogénica es producida por la afección de estructuras dentarias y periodontales. La causa principal es la caries dental no tratada a tiempo, la cual conduce a la afección pulpar; generalmente, la necrosis pulpar de la estructura dentaria involucrada. ${ }^{2}$ Se encuentra condicionada por dos factores: la extraordinaria virulencia del microorganismo que la produce y el estado inmunosuprimido del que la padece. ${ }^{3}$

Las infecciones odontogénicas son habituales en la población pediátrica, en especial, en niños menores de 6 años, y se presentan en el sexo masculino con mayor frecuencia. En este sentido, la celulitis facial ha tenido un aumento en su prevalencia en las últimas décadas. La determinación del sitio etiológico primario y los microorganismos responsables de la infección puede ser difícil, debido a la proximidad íntima de la piel, los dientes, las glándulas salivales, los senos y el conducto auditivo. La flora microbiana y el sitio de origen de una infección pueden variar y deben determinarse para que el régimen del tratamiento más eficaz pueda comenzarse. La celulitis facial odontogénica en niños es similar a la del adulto, aunque, en el paciente pediátrico, se tendrán consideraciones especiales, ya que presenta una evolución aguda por la facilidad de diseminarse a planos profundos. ${ }^{4}$

El sistema inmunológico local de la mucosa bucal tiene un papel protagónico en los procesos de defensa del organismo. Un gran número de células mononucleares immunocompetentes han sido encontradas en las superficies de la mucosa bucal. Los linfocitos B y las células plasmáticas, con la ayuda de algunos linfocitos $\mathrm{T}$, localmente sintetizan inmunoglobulinas: IgA, IgE y pequeñas cantidades de IgG e IgM, de manera independiente del sistema inmunológico sistémico. Estas inmunoglobulinas son parte de la primera línea de defensa en la mucosa bucal. ${ }^{5}$

Ante una agresión microbiana a los tejidos, existen mecanismos inespecíficos que generan una respuesta inflamatoria. Estos consisten en la liberación de mediadores, cambios vasculares (vasodilatación y vasopermeabilidad), exudado celular, activación de leucocitos, principalmente, de polimorfonucleares. Cuando esta reacción inflamatoria continúa, aparecen células mononucleares (monolitos y linfocitos) y la subsiguiente formación de tejido de granulación. ${ }^{5,6}$

En el proceso inflamatorio, puede complejizarse el cuadro con dependencia de la magnitud de la agresión microbiana y la capacidad defensiva del organismo. Esto puede generar una respuesta inflamatoria sistémica que conduce, en ocasiones, al shock séptico, a la falla multiorgánica $y$, finalmente, a la muerte. ${ }^{6}$

\section{Microorganismos implicados en la celulitis facial odontogénica}

Si se dan las condiciones favorables (determinadas situaciones metabólicas, lesión mucosa, inmunosupresión, desequilibrio del ecosistema microbiano, etc.), los microorganismos comensales orales pueden convertirse en patógenos oportunistas. Por lo tanto, las bacterias que se aíslan en la infección odontogénica son las mismas que componen la microbiota. ${ }^{6}$

Las infecciones de la cavidad bucal pueden ser bacterianas, micóticas o virales. Las infecciones odontogénicas tienden a ser mixtas, anaerobias y aerobias, a pesar de que los microorganismos anaerobios predominan..$^{7-10}$

Los microorganismos más frecuentemente implicados son Gram (+) aerobios facultativos de tipo estreptococo y Gram (-) estrictos, como Prevotella, porfiromonas y fusobacterias. ${ }^{11}$

Los agentes bacterianos que con mayor frecuencia están implicados en las infecciones odontogénicas son el Streptococcus mutans $(24,5 \%)$, Porphyromonas gingivalis (23,6\%), Porphyromonas endodontalis $(18,2 \%)$. Debido a sus factores virulentos, al tipo de metabolismo bacteriano y a los requerimientos nutricionales del paciente, se convierten en los agentes causales más importantes el Streptococcus salivarius $(10,1 \%)$ y el Streptococcus sanguis $(8,2 \%))^{12,13}$

Por su parte, Leitao, Pedemonte, Basili, en su estudio de acción microbiana en niños y adultos, encontraron que las infecciones bucofaciales derivadas de necrosis pulpar en ambos grupos 
eran diferentes. Reportaron que las infecciones por aerobios resultaron ser significativamente mayores en los niños (20\%), en comparación con los adultos $(10 \%)$, por anaerobios en adultos $(40 \%)$ e inexistente en niños $(0 \%)$ y que las infecciones mixtas eran predominantes en ambos grupos (niños: 75\%; adultos: $45 \%$ ). ${ }^{12-14}$

Otro de los microorganismos implicados en la celulitis facial de origen dental en niños es el Streptococcus pyogenes, en ocasiones, asociado a otros microorganismos presentes en la flora bacteriana bucal. ${ }^{15}$

Entre los factores que influyen la diseminación de la infección, se encuentran los siguientes:

\section{Factores generales}

- Disminución de la inmunidad: la proliferación y diseminación bacteriana se hacen más rápidas en el caso de pacientes sistémicamente comprometidos (ejemplo: diabéticos no controlados), en los cuales la resistencia se encuentra disminuida, aunque la cantidad de microorganismos no sea alta y su virulencia no sea significativa. ${ }^{16}$

- La virulencia: está dada por las cualidades del microorganismo, que favorecen la invasividad, mediante la producción de enzimas líticas, endotoxinas y exotoxinas. ${ }^{16}$

- La cantidad de bacterias: es importante, ya que, en el foco inicial, influye en la magnitud de la infección; simultáneamente, aumenta la capacidad de vencer los mecanismos de defensa del huésped y se eleva la concentración de productos tóxicos. ${ }^{16-18}$

\section{Factores locales}

- El hueso alveolar constituye la primera barrera de tipo local. La infección se disemina de manera radial y se relaciona posteriormente con el periostio, el cual es más desarrollado en el hueso mandibular que en el maxilar. En la mayoría de los casos, la infección se extiende a los tejidos blandos subyacentes; la disposición anatómica de los músculos y aponeurosis determina el siguiente sitio de localización. ${ }^{16}$

Posibles complicaciones si la celulitis facial odontogénica no es tratada de forma adecuada

La mayor parte de las infecciones odontogénicas suelen tratarse en forma adecuada con pocas complicaciones; sin embargo, si se diseminan, provocan complicaciones graves e incluso la muerte. ${ }^{8}$

Entre las complicaciones, se encuentran sinusitis odontogénica, infecciones periorbitarias, trombosis del seno cavernoso, endocarditis bacteriana, angina de Ludwig, fascitis necrosante cervicofacial, absceso cerebral, meningitis, mediastinitis, septicemia, encefalitis gangrenosa, neumonía gangrenosa, tromboflebitis de las venas yugulares, edema de glotis. ${ }^{17,18}$

Las complicaciones de esta patología dependerán directamente del cuadro general del paciente, del sistema inmunológico y de la presencia o no de una enfermedad sistémica, que agravaría el proceso. La celulitis facial odontogénica en niños se caracteriza por la rapidez del proceso séptico inducida por la amplitud de los espacios medulares, que genera riesgo vital. Puede ocasionar convulsiones, fiebre elevada con daño cerebral. El paciente puede presentar un cuadro de deshidratación marcada por las características propias del niño. Además, se pueden afectar los centros de crecimiento esqueletal. ${ }^{18}$

Los pacientes deben ser remitidos a un centro hospitalario para recibir cuidados médico-quirúrgicos especializados cuando se incluya alguno de los siguientes criterios: celulitis de progreso rápido, disnea, disfagia, extensión a espacios profundos, fiebre mayor de $38{ }^{\circ} \mathrm{C}$, trismus intenso de la articulación temporomandibular (ATM), pacientes no colaboradores o incapaces de seguir por sí mismos el tratamiento ambulatorio, fracaso del tratamiento inicial, afección grave al estado general y pacientes inmunocomprometidos. ${ }^{12,19}$

Han sido reportados casos de pacientes que, por infecciones odontogénicas difusas, llevan a sepsis, que incluyen la región del mediastino y la afección hepática en quienes sufren de enfermedades inmunodepresoras. ${ }^{20,21}$

En un paciente pediátrico, existen casos en los que la infección no es capaz de restringirse a los tejidos locales y se disemina a lo largo de la vía de menor resistencia, por lo que afecta, en primer lugar, a espacios maxilofaciales superficiales y puede llegar a comprometer espacios anatómicos profundos. Por tal motivo, se hace necesario el conocimiento por parte del personal médico y odontológico del manejo de las infecciones odontogénicas en niños para evitar, en lo posible, alguna de las complicaciones. ${ }^{22}$

Dentro de las complicaciones, existen algunas que representan un mayor riesgo letal para el paciente, como la trombosis del seno cavernoso, la cual puede estar causada por un proceso infeccioso o no infeccioso. ${ }^{22}$

En las infecciones graves del mediastino, solo un diagnóstico y tratamiento oportuno 
puede reducir la alta tasa de mortalidad de esta enfermedad. La mediastinitis necrosante es causada, principalmente, por infecciones orofaríngeas, en especial, por infecciones de origen odontogénico..$^{23-26}$

Otra de las complicaciones graves es la fascitis necrotizante por Streptococcus pyogenes, la cual representa una emergencia quirúrgica con una elevada tasa de mortalidad infantil, por la rápida progresión a shock y falla multiorgánica. El reto en esta patología supone realizar un diagnóstico precoz, que suele ser confundida con una infección leve de tejido blando. ${ }^{27}$

Por último y no menos importante, dentro de las complicaciones graves, se encuentran las infecciones periorbitarias, que pueden originarse por un proceso infeccioso en los maxilares y diseminarse en forma directa, aunque también pueden producirse por vía hematógena. La órbita representa una estructura anatómica parcialmente cerrada con paredes delgadas, ausencia de sistema linfático profundo y un sistema venoso sin válvulas. Una infección orbitaria mal diagnosticada y mal tratada puede dar origen a la trombosis del seno cavernoso, extenderse y producir tromboflebitis de las venas vecinas, lo que ocasiona sensibilidad del globo ocular ante la presión, hipertermia, dolor ocular, ptosis palpebral, etc. ${ }^{28-30}$

\section{Atención multidisciplinaria de la celulitis facial odontogénica en niños}

El tratamiento de la celulitis facial odontogénica en niños está en relación con las condiciones del estado general del paciente y el estado evolutivo del cuadro clínico. Para obtener un tratamiento y resolución del cuadro infeccioso, es necesaria la elaboración de la historia clínica, con el correcto diagnóstico de la patología, identificando el agente causal, en este caso, identificando la unidad dentaria que es considerada el foco de infección principal. Se deben emplear los respectivos métodos diagnósticos, como la radiografía periapical, la radiografía panorámica, el examen clínico extrabucal e intrabucal del paciente, la evaluación de las condiciones generales del paciente $y$, en algunos casos, se amerita la tomografía axial computarizada y la ecografía de partes blandas. ${ }^{1,31}$

No todas las infecciones odontogénicas requieren terapia antimicrobiana: depende de las características del proceso infeccioso. Generalmente, cuando un niño presenta una celulitis odontogénica, los padres, en un alto porcentaje, desconocen la causa etiológica; por tal motivo, es trasladado a un centro de salud donde se realizan todos los exámenes de diagnóstico correspondientes para determinar la causa de la infección. ${ }^{11}$

La terapéutica se basa en dos principios fundamentales: la eliminación de la causa subyacente y el drenaje local o desbridamiento y el uso de los agentes antimicrobianos por vía oral o parenteral. ${ }^{12}$

Si una infección local se deja sin tratar, puede ocurrir la propagación de las enfermedades infecciosas hacia las regiones superiores o inferiores de la cara. ${ }^{32-34}$ El niño presenta características especiales que aumentan la posibilidad de que esto suceda. Sus características anatómicas y fisiológicas generales son muy variadas debido al amplio rango etario que abarca la odontopediatría. ${ }^{35}$

Desde el punto de vista local, las características anatómicas de los maxilares infantiles también difieren. La presencia de gérmenes dentarios, la mayor cantidad de hueso esponjoso con espacios medulares amplios y la presencia de centros de crecimiento óseo hacen que la difusión de los procesos infecciosos sea más rápida que en los adultos. El odontopediatra debe tomar en cuenta la evolución de ese paciente y prestar atención a los criterios de alarma que puedan derivar en la necesidad de hospitalización. ${ }^{36}$

El manejo intrahospitalario está indicado en pacientes que tienen compromiso de más de dos espacios aponeuróticos, no toleran la vía oral y no se resuelve el cuadro infeccioso con manejo clínico ambulatorio. ${ }^{1,35}$

Se establecen como criterios para la hospitalización de un niño o adolescente con una celulitis odontogénica los siguientes:

- Afectación del estado general y/o paciente inmunocomprometido, como puede ser un paciente diabético, con malnutrición, con virus de la inmunodeficiencia humana (VIH), etc.

- Celulitis rápidamente progresiva.

- Tiempo de evolución, generalmente, más de 24 horas.

- Celulitis que se extiende a espacios faciales profundos.

- Compromiso de la vía área superior (espacio laterofaríngeo, pretraqueal, retrofaríngeo).

- Toque general del paciente con fiebre superior a $38^{\circ} \mathrm{C}$, presencia de disnea y / o disfagia y/o trismus intenso que limita la apertura bucal a menos de $10 \mathrm{~mm}$.

- Paciente o familia no colaboradora o incapaz 
de seguir el tratamiento ambulatorio prescrito.

- Fracaso del tratamiento inicial.

Si, al ser evaluado el paciente odontológicamente, se determina que presenta alguno de los criterios para ser hospitalizado, se debe realizar la interconsulta con el médico pediatra para ser evaluado en dicha área, para estabilizarlo y que se restablezca su soporte médico y nutricional, la administración de líquidos y electrolitos y el inicio de terapia antibiótica y analgésica, de acuerdo con los tejidos comprometidos. ${ }^{36}$

Odontológicamente, en las infecciones de origen odontogénico en niños, tanto en la dentición primaria como en la permanente joven, el tratamiento local es prioritario. Este consiste en la apertura cameral (drenaje dentario) del diente involucrado, con o sin la limpieza de los conductos, para permitir la salida o drenaje del contenido purulento, si lo hubiese. Si el tratamiento local no se realiza y únicamente se administran antimicrobianos, se estará disminuyendo la virulencia del proceso, que se reagudizará al suspender la medicación. ${ }^{37}$

Luego de solucionar el cuadro agudo de la infección odontogénica, se decide si el tratamiento definitivo es la conservación de la unidad dentaria mediante una pulpectomía no vital más la restauración definitiva o la exodoncia; esto dependerá del estudio radiográfico de la unidad dentaria. ${ }^{37}$

Históricamente, los betalactámicos constituyeron los agentes de primera línea en los tratamientos de las infecciones odontogénicas. La penicilina $G$ cristalina (parenteral) y la penicilina $\mathrm{V}$ (oral) son altamente eficaces, ya que gran parte de las bacterias son sensibles a ellas; sin embargo, los géneros Prevotella, Bacteroides y otros microorganismos son productores de enzimas resistentes a penicilinas. En este escenario, las aminopenicilinas asociadas a inhibidores (amoxicilina-ácido clavulánico, ampicilinasulbactam) constituyen la primera opción terapéutica en la mayoría de las infecciones de origen dental y sus complicaciones, siempre y cuando el paciente no presente hipersensibilidad a la penicilina. ${ }^{3}$

Existe otra clasificación de ingreso hospitalario de las infecciones odontogénicas en niños y adolescentes:

1. Infecciones odontogénicas leves: amoxicilina + ácido clavulánico por vía oral. ${ }^{38}$

2. Celulitis y flemones faciales: amoxicilina + ácido clavulánico por vía oral (si no hay respuesta a través de la vía oral o existe una rápida progresión de la infección, se trata de forma alternativa con clindamicina intravenosa). ${ }^{38}$

3. Celulitis faciales graves: amoxicilina + ácido clavulánico intravenoso a razón de $100 \mathrm{mg} /$ $\mathrm{kg} /$ día.

En algunos países, no hay presentación en solución oral para la clindamicina, lo que representa un inconveniente en odontopediatría y en el área de pediatría; por tal motivo, en estos casos, se indica la administración por vía parenteral de este antibiótico si el paciente presenta hipersensibilidad a la penicilina. ${ }^{37,38}$

\section{CONCLUSIONES}

Establecer un diagnóstico precoz y correcto de las infecciones faciales odontogénicas por parte del pediatra de atención primaria, el correcto uso de antibióticos y la realización del tratamiento odontológico son clave para la resolución rápida de esta afección. Fundamentalmente, deben remitirse para considerar su ingreso los pacientes con celulitis facial odontogénica de progresión rápida, con trismus, afectación general o fracaso del tratamiento antibiótico por vía oral. El diagnóstico y tratamiento hospitalario a tiempo pueden evitar la aparición de complicaciones graves.

Es indispensable la presencia del odontopediatra a nivel intrahospitalario, debido a la alta prevalencia de este tipo de patologías y las complicaciones que pueden surgir si no se trata de forma adecuada.

Se recomienda investigar más sobre este tema e insistir en la higiene bucal y las revisiones odontológicas en forma periódica, que son esenciales para prevenir y tratar la caries y la enfermedad periodontal, y evitar su progreso.

\section{REFERENCIAS}

1. Batista Sánchez T, Martínez Chacón M, Rojas Escobar R et al. Celulitis facial odontógena en pacientes del Hospital Lenin de Holguín. CCM 2017;21(2):34-6.

2. Grandas Ramírez AL, Velásquez Cuchia S. Prevalencia de celulitis odontogénica en pacientes de 0 a 18 años que asistieron a la Fundación HOMI-Hospital dela Misericordia de Bogotá entre Febrero de 2009 a Febrero de 2011. Acta Odontol Col 2012; 2(1):71-85.

3. Zambrano G, Rondón R, Guerra ME. Diente más afectado por la celulitis facial de origen odontogénico en niños venezolanos. Rev Odontopedatr Latinoam 2016;6(2):81-9.

4. Kara A, Ozsurekci Y, Tekcicek M, et al. Length of hospital stay an management of facial cellulitis of odontogenic origin in children. Pediatr Dent 2014;36(1):e18-22.

5. Michael J, Hibbert S. Presentation and management of facial swellings of odontogenic origin in children. Eur Arch Paediatr Dent 2014;15(4):259-68. 
6. Lin $Y, L u P$. Retrospective study of pediatric facial cellulitis of odontogenic origin. Pediatr Infect Dis J 2006;25(4):339-42.

7. Wang J, Ahani A, Pogrel MA. A five-year retrospective study of odontogenic maxillofacial infections in a large urban public hospital. Int J Oral Maxillofac Surg 2005;34(6):646-9.

8. García Villarmet C, De la Teja Ángeles E, Ceballos Hernández $\mathrm{H}$, et al. Infecciones faciales odontogénicas: Informe de un caso. Acta Pediatr Mex 2007;28(3):95-100.

9. Unkel JH, McKibben DH, Feston SJ, et al. Comparision of odontogenic and nonodontogenic facial cellulitis in a pediatric hospital population. Pediatr Dent 1997;19(8):476-9.

10. De la Teja AE, Escudero CA, Elías MG. Infecciones Odontogénicas. Acta Pediatr Mex 1999;4(5):208-10.

11. Gutiérrez Pérez JL, Perea Pérez EJ, Romero Ruiz M, et al. Infecciones orofaciales de origen odontogénico. Med Oral Patol Oral Cir Bucal (Ed Impr) 2004;9(4):280-7.

12. Acosta MG, Bolívar M, Nuñez N. Manejo de infecciones odontogénicas en niños. Revisión de la literatura. Rev AMOP 2017;29(2):52-8.

13. Leitao A, Pedemonte T, Basili E. Microorganismos predominantes en abscesos odontologicos de adultos y niños. Rev Dent Chile 2004;95(2):29-33.

14. Domínguez Guerra S, Machín Hernández A, Paredes Suárez M, et al. Comportamiento de la celulitis cérvicofacial en una comunidad venezolana. Rev Ciencias Médicas 2012;16(2):78-81.

15. Sánchez Saldaña L. Celulitis facial odontogénica en niños. Dermatol Peru 2012;23(2):72.

16. Chura N. Celulitis cervicofacial de origen dentario. Rev Act Clin Med 2010;1(3):40-5.

17. Salazar M. Infecciones osteoarticulares y de piel. En González SN, Torales TA, Gómez BD. Infectología clínica pediátrica. $7{ }^{\mathrm{ma}}$ ed. México: McGraw-Hill Interamericana; 2004.Págs.3-31.

18. Maestre JR. Infecciones bacterianas mixtas de la cavidad oral. Enferm Infecc Microbiol Clin 2002;20(2):98-101.

19. De Vicente-Rodríguez JC. Celulitis maxilofaciales. Med Oral Patol Oral Cir Bucal 2004;(9 Suppl):S126-38.

20. Juncar M, Popa AR, Lung T, et al. Septic metástasis of supuration of odontogenic origin. Chirurgia (Burcur) 2011;106(3):359-64.

21. Azenha MR, Homsi G, García IR Jr. Multiple brain abscess from dental origin: case report and literatura review. Oral Maxillofac Surg 2012;16(4):393-97.

22. Navazo Eguía A, Clemente García A, García Vicario F, et al. Tromboflebitis del seno cavernoso. A propósito de un caso. Rev Soc Otorrinolaringol Castilla León Cantab La Rioja 2010;1(16):1-8.

23. Pérez RA, Cueto RG, Escosura RG, et al. Mediastinitis necrosantedescendente. Resultados del tratamiento médicoquirúrgico en 17 casos. Gac Med Mex 2003;139(3):199-204.

24. Adelsdorfer Orellana C, Zuñiga González C, Díaz Díaz $\mathrm{P}$, et al. Manejo agresivo en mediastinitis necrotizante descendente. Rev Chil Cir 2005;57(3):245-50.

25. Lizardo-Castro G, García D. ¿Qué debemos saber hoy en día de la fascitis necrotizante? A propósito de 2 casos. Rev Med Hondur 2011;79(4):203-7.
26. Comité Nacional de Infectología. Infecciones de piel y partes blandas en pediatría: consenso sobre diagnóstico y tratamiento. Arch Argent Pediatr 2014;112(1):96-102.

27. Rodríguez Lorenzo A, Midón Míguez J, Martelo Villar F. Síndrome del shock tóxico estreptocócico tras fascitis necrotizante por estreptococo pyogenes. Cir Plast Iberolatinoam 2007;33(4):257-62.

28. La Orden Izquierdo E, Ruiz Jiménez M, Blázquez Fernández JA, et al. Revisión de celulitis periorbitaria y orbitaria. Experiencia de 15 años. Rev Pediatr Aten Primaria 2009;11(44):597-606.

29. Jiménez Y, Bagán JV, Murillo J, et al. Infecciones Odontogénicas. Complicaciones. Manifestaciones sistémicas. Med Oral Patol Oral Cir Bucal 2004;9 Suppl:S139-47.

30. Rodríguez Calzadilla M, García Vázquez M, Contreras Espuny $\mathrm{M}$, et al. Enfisema periorbitario secundario a procedimiento dental: reporte de un caso revisión de la literatura. Rev Mex Oftalmol. 2017. [Acceso: 12 de enero de 2018]. Disponible en: https://www.sciencedirect.com/ science/article/pii/S0187451917300859.

31. Medina Vega LD, Martín Pino J, Portal Fernández W, et al. Utilización de la moxibustion en el tratamiento de la celulitis facial odontogénica. Acta Odontol Venez 2006;44(1):65-9.

32. Maestre Vera JR. Opciones terapéuticas en la infección de origen odontogénico. Med Oral Patol Oral Cir Bucal 2004;(9 Suppl):S19-31.

33. Dodson TB, Perrott DH, Kaban LB. Pediatric maxillofacial infections: A retrospective study of 113 patients. J Oral Maxillofac Surg 1989;47(4):327-30.

34. Fine DH, Hammond BF, Loesche WJ. Clinical use of antibiotics in dental practice. Int J Antimicrob Agents 1998;9(4):235-8.

35. Caviglia I, Techera A, García G. Terapias antimicrobianas en infecciones odontogénicas en niños y adolescentes. Revisión de la literatura y recomendaciones para la clínica. Odontoestomatología 2016;18(27):46-9.

36. Bascones Martínez A, Aguirre Urízar JM, Bermejo Fenoll A, et al. Documento de consenso sobre el tratamiento antimicrobianodelasinfecciones bacterianas odontogénicas. Med Oral Patol Oral Cir Bucal 2004;9(5):363-76.

37. Nishimura RA, Carabello BA, FaxonDP, et al. ACC/AHA 2008 guideline update on valvular heart disease: focused update on infective andocarditis: a report of the American Collegue of Cardiology / American Heart Association Task Force on Practice Guidelines endorsed by the Society of Cardiovascular Anesthesiologists, Society Cardiovascular Angiography and Interventions, and Society of Thoracic Surgeons. J Am Coll Cardiol 2008;52(8):676-85.

38. Costales González C, Zafra Anta MA, Alfaro Iznaola C, et al. Ingresos por celulitis facial odontogénica en un hospital deMadrid.Características clínicas y tratamiento. Act Pediatr Esp. 2015;73(7):e158-64. 